Copyright by the IOP PUBLISHING LTD. D. A. Boboltz et al. 1997. "R Aquarii: First Detection of Circumstellar SiO Maser Proper Motions," ApJ 487 L147 doi:10.1086/310896

The Astrophysical Journal, 487:L147-L150, 1997 October 1

No copyright is claimed for this paper. Printed in U.S.A.

\title{
R AQUARII: FIRST DETECTION OF CIRCUMSTELLAR SIO MASER PROPER MOTIONS
}

\author{
D. A. Boboltz, ${ }^{1,2}$ P. J. Diamond, ${ }^{1}$ And A. J. Kemball ${ }^{1}$ \\ Received 1997 May 21; accepted 1997 July 18; published 1997 September 2
}

\begin{abstract}
We have made the first detection of circumstellar $\mathrm{SiO}$ maser proper motions in the envelope of a late-type star. Using the Very Long Baseline Array (VLBA), we have obtained observations at four epochs of the $43 \mathrm{GHz}$, $v=1, J=1-0 \mathrm{SiO}$ maser emission toward the Mira variable in the symbiotic binary $\mathrm{R}$ Aqr. The maser emission has a ringlike structure approximately 31 mas across with a slight elongation in the north-south direction. We find that the emission changes significantly over a timescale of about 1-2 months with almost no similarity in structure for timescales $\geq 6$ months. Our observations show that over a 98 day period the masers have an average inward proper motion of about 1 mas. This contraction of the ring implies an infall velocity of about $4 \mathrm{~km} \mathrm{~s}^{-1}$ for the $\mathrm{SiO}$ masers in the circumstellar envelope.
\end{abstract}

Subject headings: binaries: symbiotic — circumstellar matter — masers — stars: individual (R Aquarii)

\section{INTRODUCTION}

Astronomical maser emission provides a unique probe of the kinematics and dynamics of the circumstellar envelopes of latetype stars. Many of these stars exhibit maser emission from one or more molecular species, including $\mathrm{OH}, \mathrm{H}_{2} \mathrm{O}$, and $\mathrm{SiO}$. The conditions necessary for the formation of $\mathrm{SiO}$ masers dictate that they be produced in a region that is inside the silicate dust formation point close to the surface of the star. This extended atmosphere is a complex region dominated by stellar pulsations and permeated by circumstellar shocks. $\mathrm{SiO}$ maser emission toward long-period variables such as R Aqr allows the observer (through multiepoch experiments) to study the time evolution of this environment over a single stellar pulsation cycle.

$\mathrm{R}$ Aqr is the closest known symbiotic binary at a distance of about $220 \mathrm{pc}$ (van Belle et al. 1996). This D-type symbiotic system consists of a $\sim 1.5 M_{\odot}$ Mira variable and an unseen $\sim 1$ $M_{\odot}$ hot companion that accretes matter through a disk. The long-period variable is of spectral type M7 with a 387 day pulsation period. The orbital period of the binary is uncertain, with the best estimate being about $44 \mathrm{yr}$ (Willson, Garnavich, $\&$ Mattei 1981). The system possesses a well-studied astrophysical jet, first detected through optical observations (Herbig 1980; Wallerstein \& Greenstein 1980) and subsequently observed at radio frequencies (Sopka et al. 1982).

$\mathrm{R}$ Aqr is also one of only two symbiotic binaries (the other being H1-36 Arae) that exhibit maser emission. SiO maser emission toward R Aqr was first detected by Lepine, LeSqueren, \& Scalise (1978). Since then, single-dish observations have been made of the $v=1, J=1-0$ and $v=2, J=1-0$ transitions at $43 \mathrm{GHz}$, the $v=1, J=2-1$ transition at $86 \mathrm{GHz}$, and most recently (Gray et al. 1995) the $v=1, J=7-6$ and $v=2, J=7-6$ transitions at $300 \mathrm{GHz}$. Connected-element radio interferometers have also been used to observe the 22 $\mathrm{GHz}, 6_{16}-5_{23} \mathrm{H}_{2} \mathrm{O}$ transition (Ivison, Seaquist, \& Hall 1994, 1995) and the $v=1, J=2-1 \mathrm{SiO}$ transition (Hollis et al. 1990) toward R Aqr. However, high-resolution very long baseline interferometry (VLBI) observations of the $\mathrm{SiO}$ maser emission have been unavailable until now.

\footnotetext{
${ }^{1}$ National Radio Astronomy Observatory, Soccoro, NM 87801.

${ }^{2}$ Virginia Polytechnic Institute and State University, Blacksburg, VA 24061.
}

Other VLBI observations of the $\mathrm{SiO}$ masers toward the latetype stars U Her, TX Cam (Diamond et al. 1994; Kemball \& Diamond 1997), W Hya (Miyoshi et al. 1994), and VX Sgr (Greenhill et al. 1995) have shown that the masers generally exhibit a ringlike morphology and are located a few stellar radii from the center of the star, inside the dust formation point. In this Letter, we present multiepoch VLBI observations of the $\mathrm{SiO}$ emission toward R Aqr that verify the ring structure found for other late-type stars. In addition, we are able to identify and track individual components to determine proper motions in the shell of maser emission. In $\S 2$ we discuss the observations and data reduction. The results and discussion are presented in $\S \S 3$ and 4 , respectively.

\section{OBSERVATIONS}

The $v=1, J=1-0 \mathrm{SiO}$ maser emission toward R Aqr was observed at four epochs using the Very Long Baseline Array (VLBA), a facility of the National Radio Astronomy Observatory (NRAO) ${ }^{3}$ The data were recorded in dual circular polarization in a $4 \mathrm{MHz}\left(27.8 \mathrm{~km} \mathrm{~s}^{-1}\right)$ band centered on a velocity of $-27.0 \mathrm{~km} \mathrm{~s}^{-1}$ with respect to the local standard of rest (LSR). A line rest frequency of $43.122027 \mathrm{GHz}$ was adopted. System temperatures and point-source sensitivities for each VLBA antenna in this frequency band are on the order of approximately $150 \mathrm{~K}$ and $11 \mathrm{Jy} \mathrm{K}^{-1}$, respectively.

The data were recorded in VLBA format and correlated in Socorro, New Mexico. The Astronomical Image Processing System (AIPS), maintained by NRAO, was used to analyze the 128 channel autocorrelation and cross-correlation spectra with channel spacings of $31.25 \mathrm{kHz}\left(\sim 0.2 \mathrm{~km} \mathrm{~s}^{-1}\right)$. A detailed discussion of the calibration of full-polarization, VLBI spectral line data sets can be found in García-Barreto et al. (1988) and Kemball, Diamond, \& Cotton (1995). The calibration procedure used in the reduction of the polarization data for these observations will be presented in a later paper along with the polarization results. The calibration procedure used to derive the total intensity images presented in this Letter follows that described in Reid et al. (1980) and Diamond (1989) and the papers mentioned above.

\footnotetext{
${ }^{3}$ The National Radio Astronomy Observatory is a facility of the National Science Foundation operated under cooperative agreement by Associated Universities, Inc.
} 


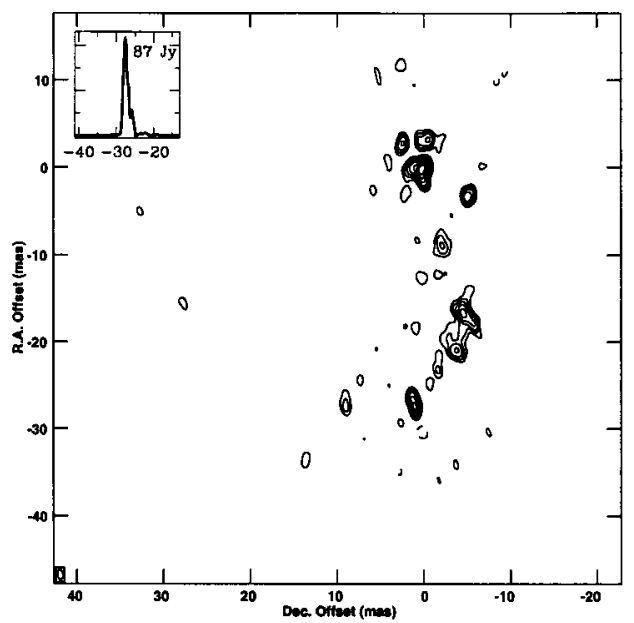

(a) Epoch 1 (30 June 1995, $\phi=0.31$ )

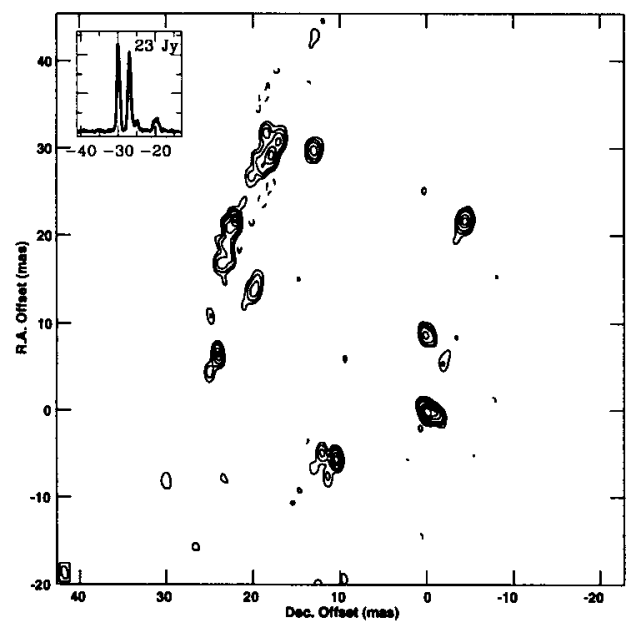

(c) Epoch 3 (2 February 1996, $\phi=0.87$ )

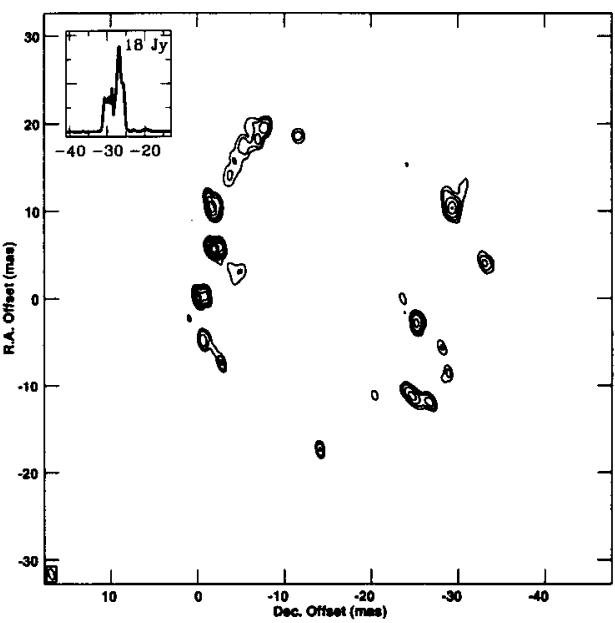

(b) Epoch 2 (29 December 1995, $\phi=0.78$ )

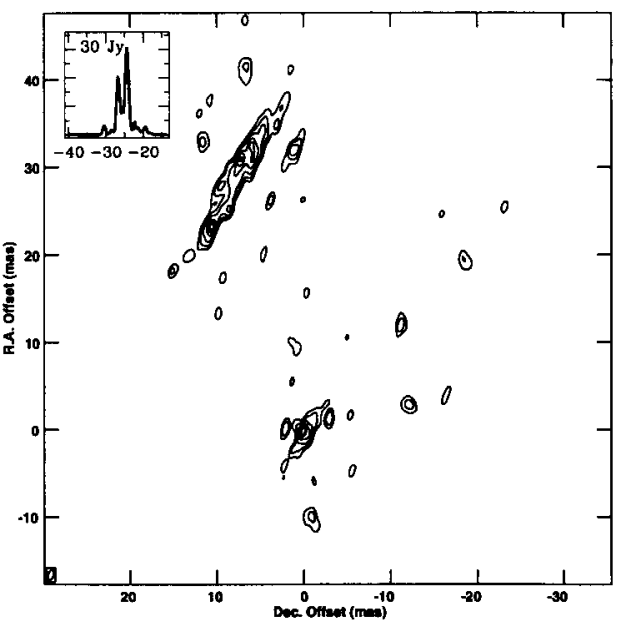

(d) Epoch 4 (5 April 1996, $\phi=0.04$ )

FIG. 1.-Total intensity VLBI images of the $v=1, J=1-0 \mathrm{SiO}$ maser emission toward R Aqr integrated over the LSR velocity range of $-32.9 \mathrm{~km} \mathrm{~s}{ }^{-1}$ to $-17.4 \mathrm{~km} \mathrm{~s}^{-1}$. Contour levels are $-10,-5,5,10,20,40,60,80$, and 100\% of the peak integrated flux density in each image. Peak integrated flux densities obtained by summing over all channels within the above velocity range are as follows: (a) $135 \mathrm{Jy} \mathrm{beam}^{-1}$, (b) $36 \mathrm{Jy} \mathrm{beam}^{-1}$, (c) $44 \mathrm{Jy}$ beam ${ }^{-1}$, and $(d) 51 \mathrm{Jy}$ beam $^{-1}$. In the top left-hand corner of each image is a corresponding cross-power spectrum. Epochs 1, 2, and 4 show the Los Alamos-Pie Town baseline, while epoch 3 shows the Kitt Peak-Los Alamos baseline.

\section{RESULTS}

\subsection{Images of the Circumstellar Shell}

Synthesis images of the $\mathrm{SiO}$ maser emission toward $\mathrm{R}$ Aqr for each of the four epochs were constructed using standard self-calibration and imaging techniques. Image cubes consisting of individual channel maps of size approximately $90 \times$ 90 mas were made for velocities between $-32.9 \mathrm{~km} \mathrm{~s}^{-1}$ and $-17.4 \mathrm{~km} \mathrm{~s}^{-1}$. The geometric mean of the synthesized beamwidth for each of the four epochs was about 0.9 mas in natural weighting.

Figure 1 shows the total intensity images integrated over frequency for each of the four epochs. A corresponding shortspacing cross-power spectrum is shown in the top left corner of each image. The optical phase of the Mira variable with respect to maximum light $\phi$ is also indicated for each epoch of observations. The absolute error in the amplitude calibration is estimated to be between 5\% and 15\%. Figure $1 a$ (epoch 1) shows an arc or partial ring of maser emission extending in the north-south direction. In epoch 2 , which was observed only 6 months later, the masers form nearly a full ring. This structure is evident in all three subsequent epochs, spanning a time period of approximately 3 months. Assuming a distance of $220 \mathrm{pc}$ to $\mathrm{R}$ Aqr, the projected ring of emission has a diameter of $\sim 6.8$ AU, with an average width (FWZP) of $\sim 0.7$ AU. The structure is slightly elongated in the north-south direction, and there appears to be a gap in the emission that has a north-northwest-south-southeast orientation. This gap could be related to the formation of the R Aqr jet, which extends in a roughly perpendicular northeast-southwest direction (Kafatos et al. 1989).

The four epochs shown in Figure 1 clearly show the strong time-variable nature of the maser emission. Epoch 1, which in general has the strongest features, appears to have few related features in epoch 2. Over the 6 month time period from epoch 1 to epoch 2, the entire eastern side of the ring appears. This is accompanied by significant changes in the western portion of the ring. New features appear on timescales as short as about 1 month, as is evident in the evolution of the southernmost feature in Figure $1 b$. This feature consists of a single maser spot in epoch 2, two distinct spots of emission in epoch 3 , and three spots of maser emission in epoch 4 . In addition to the 


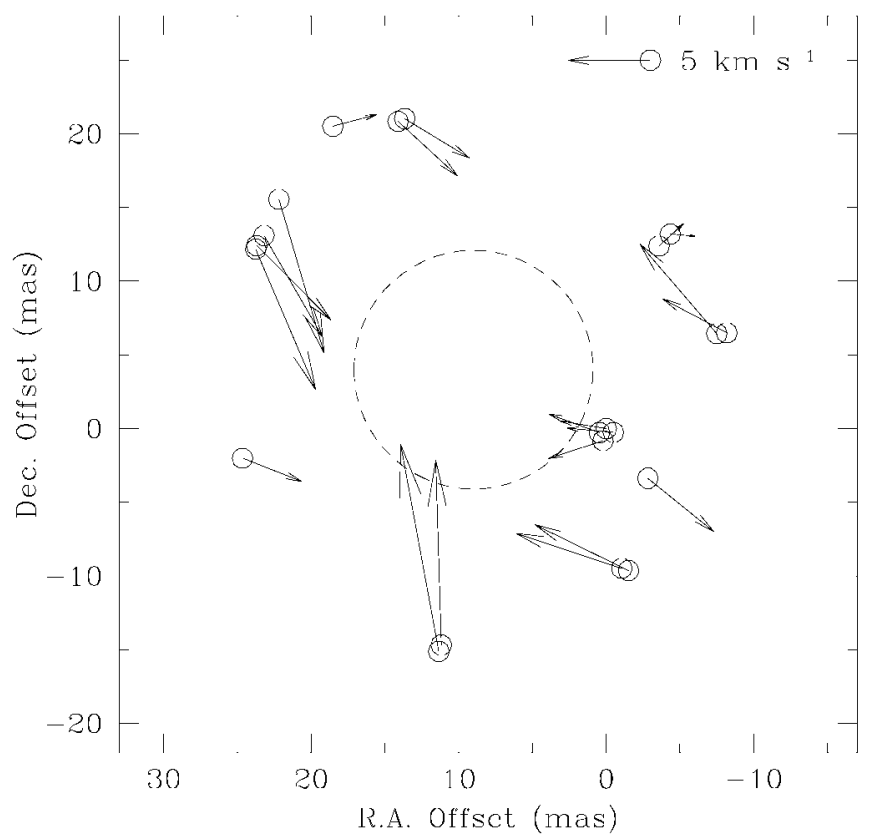

FIG. 2.-Map representing the distribution of proper-motion velocity vectors from epoch 2 to epoch 4 at the assumed distance of $220 \mathrm{pc}$. Proper motions are uncertain by an absolute velocity vector representing the motion of the feature used to align the three epochs. The mean proper-motion vector has been subtracted from all components. The dashed circle in the center represents a stellar disk with a diameter of $3.6 \mathrm{AU}$ at the adopted distance.

formation of new features, existing features also vary with time. The structure of the northeast section of the ring, which is made up of several individual features in epoch 2, changes significantly over 98 days, appearing as a linear filamentary structure by epoch 4 . The components comprising the western side of the ring are relatively strong in epochs 2 and 3 but are much weaker in epoch 4 .

\subsection{Proper-Motion Determination}

From Figures $1 b-1 d$ it is apparent that some of the maser features span multiple epochs. The 6 month separation between the first and second epochs is too large to allow proper-motion determination, for reasons discussed above. To determine any proper motions of the masers in the shell, individual components were measured for the final three epochs. Features were first identified by fitting a two-dimensional Gaussian the size of the synthesized beam to potential sources in each spectral channel of the image cube. Components below 8 times the $1 \sigma$ off-source noise level or $80 \%$ of the deepest negative in the image were rejected. Component positions in right ascension, declination, and velocity were determined using a flux density squared weighted average for those identifications found in two or more channels, spatially coincident to within approximately $\frac{1}{3} \theta_{\text {beam }}$, with peak flux densities greater than 400 mJy. The $400 \mathrm{mJy}$ cutoff was chosen to be lower than the absolute value of the lowest contour believed to be significant in the individual channel maps of all four epochs.

Because of the nature of standard VLBI data reduction, the absolute position of the phase center in each image is unknown. The coordinate frames for the final three epochs were shifted to align the origin for each set of components on the same feature at $V_{\mathrm{LSR}} \approx-19.5 \mathrm{~km} \mathrm{~s}^{-1}$. Components were then matched from one epoch to another. Since the three epochs were aligned on a single component, derived proper motions are uncertain

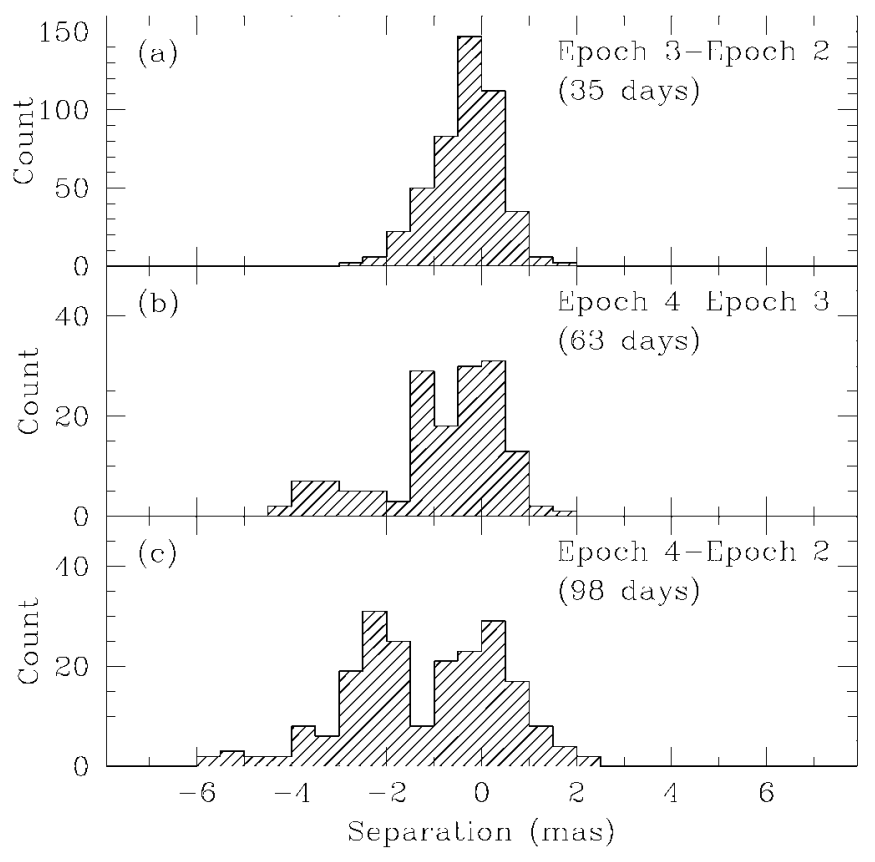

FIG. 3.-Histograms showing the change in pairwise component separations for $(a)$ epoch 2 to epoch $3,(b)$ epoch 3 to epoch 4, and (c) epoch 2 to epoch 4. All component pairs were included in the derivation of these histograms. Bias toward negative shifts indicates contraction of the maser shell.

by a constant offset vector representing the motion of that feature. In order to present a visual representation of the expansion or contraction, we subtracted the mean proper motion from each of the determined proper-motion vectors. The proper motions from epoch 2 to epoch 4 are shown in Figure 2. These proper-motion vectors indicate a general inward motion for the majority of components.

One way to test for any net expansion or contraction of the masers is to compute separations between pairwise combinations of components. This technique has been applied to both OH masers (Chapman, Cohen, \& Saika 1991; Bloemhof, Reid, \& Moran 1992; Kemball 1992) and $\mathrm{H}_{2} \mathrm{O}$ masers (Marvel 1996) and is nonparametric, since it has no dependence on the alignment of the maps or prior knowledge of the velocity field. The histograms in Figure 3 plot the results of the computation of pairwise separations using

$$
\left|\boldsymbol{r}_{b i}-\boldsymbol{r}_{b j}\right|-\left|\boldsymbol{r}_{a i}-\boldsymbol{r}_{a j}\right|, \quad i=1, n ; j=i+1, n
$$

where $\boldsymbol{r}_{a i}=\left(x_{a i}, y_{a i}\right), \boldsymbol{r}_{b i}=\left(x_{b i}, y_{b i}\right)$, and $\left(\boldsymbol{r}_{a i}, \boldsymbol{r}_{b i}\right)$ are assumed to be matched components for epochs $a$ and $b$, respectively (Kemball 1992). Figures $3 a-3 c$ show the separations over time intervals of 35, 63, and 98 days, respectively. All three histograms have centroids that are biased toward negative values. Figures $3 b$ and $3 c$ show a bimodal distribution with the peak near zero representing separations of closely spaced components. To determine a representative value for the shifts due to the proper motion, we computed pairwise separations for only those pairs separated by more than 15 mas. The mean of each of these distributions provides a value for the shift free of any bias toward zero caused by small separations. These shifts were found to increase over time with mean values $\Delta \theta_{\text {mean }}=$ $-0.5 \pm 0.1,-1.2 \pm 0.1$, and $-1.9 \pm 0.2$ mas for 35,63 , and 98 days, respectively. Errors in the shifts were approximated as $\left|\Delta \theta_{\text {mean }}-\Delta \theta_{\text {med }}\right| / 2^{1 / 2}$, where $\Delta \theta_{\text {mean }}$ and $\Delta \theta_{\text {med }}$ are the 
mean and median shifts for each distribution, respectively. We performed a linear least-squares fit to the three values of $\Delta \theta_{\text {mean }}$ in order to determine the overall infall velocity for the maser distribution. From this fit, an infall speed $v_{\text {mean }}=4.2 \pm 0.9$ $\mathrm{km} \mathrm{s}^{-1}$ was computed. The uncertainty in this velocity was calculated as $\left|v_{\text {mean }}-v_{\text {med }}\right| / 2^{1 / 2}$, where $v_{\text {mean }}$ and $v_{\text {med }}$ are the velocities determined from independent fits to the mean and median shifts, respectively.

To verify independently the inward proper motion of the maser distribution, we fitted a simple circle to the ring of emission in each of the final three epochs. A circle was chosen because it provides a scale size for the structure with the minimum number of free parameters. Using a least-squares fit to only those components found in all three epochs, we determined the representative radius for each distribution. This radius was found to decrease in time, indicating an overall contraction of the emission. The differences in radii between epochs are roughly consistent with the shifts found using the pairwise separations with $\Delta r \approx-0.5,-0.7$, and -1.1 mas for 35,63 , and 98 days, respectively. These values are dominated by systematic errors and are meant only to confirm the trend toward contraction of the shell.

\section{DISCUSSION}

Our observations show that the $\mathrm{SiO}$ masers toward $\mathrm{R}$ Aqr exhibit a ringlike structure evident in other late-type stars. This morphology is also seen in recent low-resolution observations of R Aqr conducted with the Very Large Array (VLA) by Hollis, Pedelty, \& Lyon (1997). The maser ring, which has a projected radius of about $3.4 \mathrm{AU}$, is located in a region outside the stellar photosphere and inside the circumstellar dust shell. From $2.2 \mu \mathrm{m}$ near-infrared observations, van Belle et al. (1996) estimate the radius of $\mathrm{R}$ Aqr to be approximately $385 R_{\odot}(\sim 1.8$ AU). This places the maser shell at about $1.9 R_{*}$, slightly less than the typical 2-4 $R_{*}$ for late-type stars (Diamond et al. 1994). Danchi et al. (1994) used a radiative transfer model to deduce the inner radius of the circumstellar dust shell from their 11.15 $\mu \mathrm{m}$ mid-infrared observations. They found this inner radius to be 70 mas $\left(\sim 8.6 R_{*}\right)$ for $\mathrm{R}$ Aqr. The $\mathrm{SiO}$ masers are thus well inside the dust shell and lie closer to the stellar photosphere.

The prospect of the infall of material inside the circumstellar dust shell of a Mira variable was first noted by Hinkle, Hall, \& Ridgway (1982). Through time series, near-infrared spectroscopy of $\chi$ Cygni, they derived a picture in which stellar oscillation drives an outwardly propagating wave through the photosphere between the premaximum and maximum phases of the star. This wave sets up a shock between material being accelerated outward and material falling inward from the previous cycle. By $\phi=0.1$, the shock has passed through the envelope, and material falls inward under the influence of gravity from $\phi=0.1$ to $\phi=0.8$. The numerical calculations by Bowen (1988) demonstrate that the shock passage can occur later and that the infall can extend beyond phase 0.8 for the outer portions of the extended atmosphere.

Our observations provide the first direct evidence for infall during the stellar pulsation cycle. We have determined that the shell of $\mathrm{SiO}$ maser emission, which is inside the circumstellar dust shell, contracted through phases $0.78,0.87$, and 0.04 . The contraction of the shell over the 98 day period yields an infall speed of $4.2 \pm 0.9 \mathrm{~km} \mathrm{~s}^{-1}$. Such a velocity is easily explained assuming only gravitational infall and does not require complex mechanisms such as reverse shocks. For comparison purposes, the velocity of a particle falling in from the dust shell at 15.4 $\mathrm{AU}$ to a point on the $\mathrm{SiO}$ maser shell at $3.4 \mathrm{AU}$ would be about $25 \mathrm{~km} \mathrm{~s}^{-1}$. Our measured infall velocity is also consistent with the finding of Reid \& Menten (1997) that the shock velocities in the radio photospheres of long-period variables are below $5 \mathrm{~km} \mathrm{~s}^{-1}$ at a distances beyond approximately $2 R_{*}$. While the observations presented here only provide confirmation of the infall of material during part of the stellar cycle, monitoring the $\mathrm{SiO}$ masers over the entire range of phases would be of great benefit in determining the overall motion of the gas in the extended atmosphere of the Mira variable.

D. A. B. wishes to acknowledge the support of the NRAO predoctoral fellowship program. We would like to thank Brian Dennison for helpful comments on this manuscript.

\section{REFERENCES}

Bloemhof, E. E., Reid, M. J., \& Moran, J. M. 1992, ApJ, 397, 500

Bowen, G. H. 1988, ApJ, 329, 299

Chapman, J. M., Cohen, R. J., \& Saika, D. J. 1991, MNRAS, 249, 227

Danchi, W. C., Bester, M., Degiacomi, C. G., Greenhill, L. J., \& Townes, C. H. 1994, AJ, 107, 1469

Diamond, P. J. 1989, in Very Long Baseline Interferometry: Techniques and Applications, ed. M. Felli \& R. E. Spencer (Dortrecht: Kluwer), 233

Diamond, P. J., Kemball, A. J., Junor, W., Zensus, A., Benson, J., \& Dhawan, V. 1994, ApJ, 430, L61

García-Barreto, J. A., Burke, B. F., Reid, M. J., Moran, J. M., Haschick, A. D., \& Schilizzi, R. T. 1988, ApJ, 326, 954

Gray, M. D., Ivison, R. J., Yates, J. A., Humphreys, E. M. L., Hall, P. J., \& Field, D. 1995, MNRAS, 277, L67

Greenhill, L. J., Colomer, F., Moran, J. M., Backer, D. C., Danchi, W. C., \& Bester, M. 1995, ApJ, 449, 365

Herbig, G. 1980, IAU Circ. 3535

Hinkle, K. H., Hall, D. N. B., \& Ridgway, S. T. 1982, ApJ, 252, 697

Hollis, J. M., Pedelty, J. A., \& Lyon, R. G. 1997, ApJ, 482, L85

Hollis, J. M., Wright, M. C. H., Welch, W. J., Jewell, P. R., Crull, H. E., Kafatos, M., \& Michalitsianos, A. G. 1990, ApJ, 361, 663

Ivison, R. J., Seaquist, E. R., \& Hall, P. J. 1994, MNRAS, 269, 218
Ivison, R. J., Seaquist, E. R., \& Hall, P. J. 1995, Ap\&SS, 224, 255

Kafatos, M., Hollis, J. M., Yusef-Zadeh, F., Michalitsianos, A. G., \& Elitzur, M. 1989, ApJ, 346, 991

Kemball, A. J. 1992, Ph.D. thesis, Rhodes Univ.

Kemball, A. J., \& Diamond, P. J. 1997, ApJ, 481, L111

Kemball, A. J., Diamond, P. J., \& Cotton, W. D. 1995, A\&AS, 110, 383

Lepine, J. R. D., LeSqueren, A. M., \& Scalise, E. 1978, ApJ, 225, 869

Marvel, K. B. 1996, Ph.D. thesis, New Mexico State Univ.

Miyoshi, M., Matsumoto, K., Kameno, S., Takaba, H., \& Iwata, T. 1994, Nature, 371, 395

Reid, M. J., Haschick, A. D., Burke, B. F., Moran, J. M., Johnston, K. J., \& Swenson, G. W. 1980, ApJ, 239, 89

Reid, M. J., \& Menten, K. M. 1997, ApJ, 476, 327

Sopka, R. J., Herbig, G. H., Kafatos, M., \& Michalitsianos, A. G. 1982, ApJ, 258, L35

van Belle, G. T., Dyck, H. M., Benson, J. A., \& Lacasse, M. G. 1996, AJ, 112,2147

Wallerstein, G., \& Greenstein, J. L. 1980, PASP, 92, 275

Willson, L. A., Garnavich, P., \& Mattei, J. A. 1981, Inf. Bull. Variable Stars, No. 1961 